\title{
Combined effects of nutritional status on long-term mortality in patients with non-st segment elevation myocardial infarction undergoing percutaneous coronary intervention
}

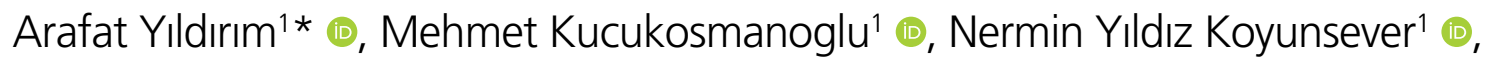

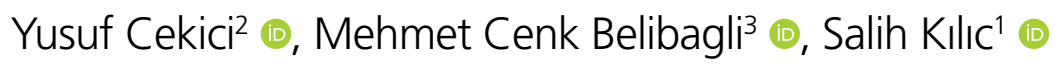

\begin{abstract}
SUMMARY
OBJECTIVE: The aim of this study was to investigate the performance of controlling nutritional status (CONUT) index, geriatric nutritional risk index (GNRI), and prognostic nutritional index (PNI) scores in predicting the long-term prognosis of patients with non-ST-elevated myocardial infarction (NSTEMI) who underwent percutaneous coronary intervention (PCI).

METHODS: A total of 915 patients with NSTEMI (female: 48.4\%; mean age: $73.1 \pm 9.0$ years) who underwent PCI at Adana Numune Training and Research Hospital, Cardiology Clinic between January 2014 and January 2015 were included in this cross-sectional and retrospective study. CONUT, GNRI, and PNI scores were calculated based on the admission data derived from samples of peripheral venous blood. The mean follow-up duration was $64.5 \pm 15.4$ months.

RESULTS: During follow-up (mean 64.5 \pm 15.4 months), 179 patients (19.6\%) died. The mean GNRI and PNI scores were significantly lower in the nonsurvivor group; however, the median CONUT score was significantly higher in the nonsurvivor group compared with the survivor group. The receiver operating characteristic (ROC) curve analyses have shown that GNRI score has similar performance to the CONUT score and has better performance than PNI score in predicting 5-year mortality. The Kaplan-Meier curve analysis has shown that patients with lower PNI or GNRI had higher cumulative mortality than the patients with higher PNI or GNRI. Also, the patients with higher CONUT scores had higher cumulative mortality compared with those with lower scores. The multivariate analyses have shown that GNRI (HR: 0.973), PNI (HR: 0.967), CONUT score (HR: 1.527), and body mass index (BMI) (HR: 0.818) were independent predictors of the 5-year mortality in patients with NSTEMI.

CONCLUSION: In this study, we have shown that CONUT score, GNRI, and PNI values were associated with the long-term mortality in patients with NSTEMI who underwent PCI, and GNRI yielded similar results to CONUT score but was better than PNI.

KEYWORDS: Controlling Nutritional Status, Geriatric Nutritional Risk Index, Prognostic Nutritional Index, Non-ST-Elevated Myocardial Infarction, Percutaneous Coronary Intervention
\end{abstract}

\footnotetext{
'University of Health Sciences, Adana Health Practice and Research Center, Department of Cardiology - Adana, Turkey. 2University of Health Sciences, Mehmet Akif İnan Research and Training Hospital, Department of Cardiology - Sanlıurfa, Turkey. 3University of Health Sciences, Adana Health Practice and Research Center, Department of Family Medicine - Adana, Turkey. *Corresponding author: arafatdr@hotmail.com Conflicts of interest: the authors declare there are no conflicts of interest. Funding: none. Received on October 15, 2020. Accepted on December 10, 2020.
} 


\section{INTRODUCTION}

Despite the evolving pharmacological treatments and reperfusion strategies, cardiovascular diseases still remain to be the leading cause of overall morbidity and mortality in the world. The majority of deaths linked to cardiovascular diseases is caused by acute coronary syndromes categorized into three conditions, namely, unstable angina pectoris, non-ST-elevated myocardial infarction (NSTEMI), and ST-elevated myocardial infarction (STEMI). Although patients with STEMI have higher in-hospital mortality, the long-term follow-up studies showed that the mortality rates increased significantly in NSTEMI cases over time ${ }^{1,2}$. Comprehensively designed studies that include the nutritional status indicate that the coexistence of cardiovascular diseases and malnutrition results in higher mortality rates ${ }^{3}$. In earlier studies, it has been shown that the malnutrition rate in hospitalized patients ranges $20-50 \%$ in developed countries and is higher in some geographical regions $s^{4,5}$. Controlling nutritional status (CONUT) index, geriatric nutritional risk index (GNRI), and prognostic nutritional index (PNI) are reliable and easily calculated nutritional indicators and have shown to have prognostic values in multiple chronic conditions ${ }^{6,7}$. In various studies, these indicators have demonstrated a strong association with the prognosis of patients with cardiovascular diseases and conditions, including coronary artery disease and congestive heart failure ${ }^{8,9}$. Among these studies, the researchers focused exclusively on acute myocardial infarction (AMI) or STEMI cases ${ }^{10,11}$. The clinical characteristics, risk factors, and prognosis of the NSTEMI differ significantly than the STEMI. The main differences are the increased mean age and the number of comorbid chronic diseases ${ }^{12}$. There are reports assessing the association of these nutritional indicators with the prognosis of NSTEMI cases exclusively. Given the noticeable differences compared with STEMI cases, the lack of a study including an analysis of CONUT score, GNRI, and PNI together may have foreclosed valuable information. The aim of this study was to investigate the performance of CONUT score, GNRI, and PNI in predicting the long-term prognosis of the patients with NSTEMI who underwent percutaneous coronary intervention (PCI).

\section{METHODS}

A total of 915 NSTEMI patients who underwent PCI at Adana Numune Training and Research Hospital, Cardiology Clinic between January 2014 and January 2015 were included in this cross-sectional and retrospective study. The diagnosis of NSTEMI was made according to the global myocardial infarction guide ${ }^{13}$. The data at the time of hospitalization, including age, gender, height, weight, body mass index (BMI), smoking, diabetes mellitus (DM), coronary artery disease, hypertension (HT), stroke, and cerebrovascular disease were collected from the patient profiles. The patients with congestive heart failure, malignancy, chronic kidney failure, nephrotic syndrome, liver failure, hematological disease, autoimmune disease, and rheumatological disease were excluded.

According to the standard criteria, admission values of patients with DM, HT, hyperlipidemia, and smoking were considered for selection. The subjects with HbA1c $>6.5 \%$, fasting blood glucose $>126 \mathrm{mg} / \mathrm{dL}$, or being on antidiabetic medication were considered as DM patients. The patients with arterial blood pressure $>140 / 90 \mathrm{mmHg}$ or being on antihypertensive medication were considered having HT. The patients with serum total cholesterol levels $>200 \mathrm{mg} / \mathrm{dL}$ or on antilipidemic medicines were regarded having hyperlipidemia. The CONUT, GNRI, and PNI scores were calculated based on the admission data derived from samples of peripheral venous blood. GNRI=14.89 ×albumin $(\mathrm{g} / \mathrm{dL})+41.7 \times$ body weight $(\mathrm{kg}) /$ ideal body weight $(\mathrm{kg})$. The ideal body weight was calculated as follows: body height-100[(body height-150)/4] for males and body height-100-[(body height-150)/2.5] for females. The CONUT scores (0-10, varying from nourishment to malnutrition) were calculated using serum albumin level and lymphocyte count. CONUT: serum albumin $\geq 3.5 \mathrm{~g} / \mathrm{dL}=0$ points, $3.0-3.4 \mathrm{~g} / \mathrm{dL}=2$ points, $2.5-2.9 \mathrm{~g} / \mathrm{dL}=4$ points, and $<2.5 \mathrm{~g} / \mathrm{dL}=6$ points; total cholesterol $\geq 180 \mathrm{mg} / \mathrm{dL}=0$ points, $140-179 \mathrm{mg} / \mathrm{dL}=1$ point, 100 $139 \mathrm{mg} / \mathrm{dL}=2$ points, and $<100 \mathrm{mg} / \mathrm{dL}=3$ points; and total lymphocyte count $\geq 1600 / \mathrm{mL}=0$ points, $1200-1599 / \mathrm{mL}=1$ point, $800-1199 / \mathrm{mL}=2$ points, and $<800 / \mathrm{mL}=3$ points (Table 1 ). PNI: $10 \times$ serum albumin value $(\mathrm{g} / \mathrm{dL})+0.005 \times$ total lymphocyte count $\left(\right.$ per $\mathrm{mm}^{3}$ ). BMI: weight (in $\mathrm{kg}$ ) $/(\text { height) })^{2}$ (in $\mathrm{m})$. As the primary end point in this study, nonsurvivor details

Table 1. Controlling nutritional status (CONUT) score calculation.

\begin{tabular}{l|c|c|c|c}
\hline Score & 0 & 2 & 4 & 6 \\
\hline Serum albumin $(\mathrm{g} / \mathrm{mL})$ & $\geq 3.5$ & $3.0-3.49$ & $2.5-2.99$ & $<2.50$ \\
\hline Score & 0 & 1 & 2 & 3 \\
\hline Total cholesterol $(\mathrm{mg} / \mathrm{dL})$ & $\geq 180$ & $140-179$ & $100-139$ & $<100$ \\
\hline Score & 0 & 1 & 2 & 3 \\
\hline Lymphocytes (count/mL) & $\geq 1600$ & $1200-1599$ & $800-1199$ & $<800$ \\
\hline
\end{tabular}


were collected from the National Health Insurance System in July 2020, including the date of death, and all causes of death were accepted.

\section{Statistical analyses}

The continuous variables were represented as mean ( \pm standard deviation) or median (25th-75th quartile). The distribution was analyzed with the Kolmogorov-Smirnov test. The Student's $t$-test was used to analyze the normally distributed variables, and the Mann-Whitney $U$ test was used for the non-normal distributions. The variables with normal distribution were represented as mean ( \pm standard deviation) and with non-normal distribution as median (25th-75th quartile).

The categorical variables were summarized as percentages and number. Categorical variables between the groups were compared by using the chi-squared test or Fisher's exact test. To demonstrate the sensitivity and specificity of the GNRI, PNI, and CONUT scores and their cut-off values for predicting the long-term mortality, the receiver operating characteristic (ROC) curves were used. The DeLong's method was used to compare area under the curve (AUC) of these nutritional indexes. The Kaplan-Meier analysis and log-rank test were performed to determine whether the nutritional indexes could help predict the long-term mortality. The multivariate Cox proportional hazards model was created to calculate the hazard ratios for all-cause mortality. Variables with $\mathrm{p} \leq 0.1$ in the univariate analysis were included in the multivariate Cox proportional hazards model.

The statistical analyses were conducted using the Statistical Package for the Social Sciences (SPSS 20.0) for Windows (SPSS Inc., Chicago, IL, USA) and MedCalc 15 statistical software (Ostend, Belgium). A $\mathrm{p}<0.05$ was considered statistically significant.

\section{RESULTS}

Totally, 915 patients (female: $48.4 \%$; mean age: $73.1 \pm 9.0$ years) were included in this study. The mean follow-up duration was $64.5 \pm 15.4$ months. During the follow-up period, 179 patients $(19.6 \%)$ died. The baseline demographic and clinical characteristics of the patients were summarized in Table 2. Despite the

Table 2. Baseline characteristics of the study population.

\begin{tabular}{l|c|c|c} 
& $\begin{array}{c}\text { Survivor Group } \\
(\mathrm{n}=736)\end{array}$ & $\begin{array}{c}\text { Nonsurvivor Group } \\
(\mathrm{n}=179)\end{array}$ & $\mathrm{p}$ \\
\hline Age, year $($ mean \pm SD) & $72.8 \pm 9.4$ & $74.1 \pm 7.1$ & 0.084 \\
\hline Sex/female, \% (n) & $47.8(352)$ & $51.4(92)$ & 0.391 \\
\hline BMI $\left(\mathrm{kg} / \mathrm{m}^{2}\right)$ & $25.6 \pm 2.6$ & $23.7 \pm 2.0$ & $<0.001$ \\
\hline Weight $(\mathrm{kg})$ & $71.4 \pm 8.2$ & $70.1 \pm 7.4$ & 0.055 \\
\hline Height $(\mathrm{m})$ & $1.67 \pm 7.2$ & $1.77 \pm 8.1$ & $<0.001$ \\
\hline SBP $(\mathrm{mmHg})$ & $125 \pm 18$ & $131 \pm 19$ & $<0.001$ \\
\hline DBP $(\mathrm{mmHg})$ & $78 \pm 10$ & $78 \pm 11$ & 0.638 \\
\hline Hypertension, \% (n) & $51.0(375)$ & $55.9(100)$ & 0.238 \\
\hline Diabetes mellitus, \% (n) & $32.3(238)$ & $33.0(59)$ & 0.873 \\
\hline Hyperlipidemia, \% (n) & $33.2(244)$ & $31.8(47)$ & 0.738 \\
\hline Stroke, \% (n) & $3.0(22)$ & $4.5(8)$ & 0.319 \\
\hline COPD, \% (n) & $7.2(53)$ & $3.9(7)$ & 0.130 \\
\hline Smoker, \% (n) & $36.0(265)$ & $34.6(62)$ & 0.732 \\
\hline Family history, \% (n) & $30.7(226)$ & $26.3(47)$ & 0.243 \\
\hline Previous myocardial infarction, \% (n) & $22.3(164)$ & $201 .(36)$ & 0.529 \\
\hline AF, \% (n) & $7.2(52)$ & $7.3(13)$ & 0.977 \\
\hline ASA, \% (n) & $20.1(148)$ & $22.9(41)$ & 0.407 \\
\hline ACEl/ARB, \% (n) & $32.1(236)$ & $28.5(51)$ & 0.355 \\
\hline Beta-blockers, \% (n) & $17.1(126)$ & $19(34)$ & 0.544 \\
\hline Diuretics, \% (n) & $5.2(38)$ & $7.8(14)$ & 0.168 \\
\hline Statins, \% (n) & $18.1(133)$ & $21.2(38)$ & 0.331 \\
\hline
\end{tabular}

ACEI: angiotensin-converting enzyme inhibitors; ASA: acetylsalicylic acid; ARB: angiotensin receptor blockers; BMI: body mass index; CONUT: controlling nutritional status; GNRI: geriatric nutritional risk index; PNI: prognostic nutritional index; COPD: chronic obstructive pulmonary disease; AF: atrial fibrillation; SBP: systolic blood pressure; DBP: diastolic blood pressure. Statistically significant values are given in bold. 
significantly higher mean age, height, and systolic blood pressure values in the nonsurvivor group, the survivor group displayed a higher mean BMI. The laboratory parameters of the two groups were summarized in Table 3 . The median total cholesterol and mean albumin levels were significantly lower in the nonsurvivor group than the survivor group. The mean GNRI and PNI values were significantly lower in the nonsurvivor group; however, the median CONUT scores were significantly higher in the nonsurvivor group compared with the survivor group (Table 3). Figure 1 shows the ROC curve analyses of the scores. To predict mortality, the cut-off value for the PNI was $\leq 50.65$, with $73.7 \%$ sensitivity and $69.4 \%$ specificity (AUC $0.714 ; 95 \% \mathrm{CI} 0.684-0.744 ; \mathrm{p}<0.001)$, the cut-off value for the GNRI was $\leq 114.77$, with $81.0 \%$ sensitivity and $65.27 \%$ specificity (AUC 0.778; 95\%CI 0.750-0.805; $\mathrm{p}<0.001$ ), and the cut-off value for the CONUT score was $>3$, with $63.1 \%$ sensitivity and $76.0 \%$ specificity (AUC $0.751 ; 95 \%$ CI $0.722-0.779$; $\mathrm{p}<0.001)$. The comparison of the ROC curve analyses shown that GNRI has similar performance to the CONUT score and has better performance than PNI in predicting 5 years mortality. Also, in predicting 5 years mortality, the prognostic values of the PNI and the CONUT scores were found similar (Figure 1).
The Kaplan-Meier curve analysis was performed for each nutritional index according to the cut-off value for all-cause mortality (Figure 2). Patients with lower PNI or GNRI had higher cumulative mortality than those with higher PNI or GNRI (37.0 vs. $8.4 \%, \mathrm{p}<0.001 ; 36.2$ vs. $6.6 \%, \mathrm{p}<0.001$, respectively). Patients with higher CONUT scores had higher cumulative mortality compared with those with lower scores ( 10.6 vs. $39.0 \%, \mathrm{p}<0.001)$. Moreover, the cumulative 5 -year mortality was higher in patients with the lowest quartile of GNRI $(\leq 107.4)$ or PNI $(\leq 46.43)$ than in patients with the top quartile of GNRI ( $\geq 125.1)$ or PNI (57.36). Furthermore, the cumulative 5-year mortality was higher in patients with the top quartile of CONUT score $(\geq 4.0)$ than in patients with the lowest quartile $(\leq 2.0)$ (Figure 3$)$. The univariate and multivariate Cox proportional hazard analyses of predictors on mortality were summarized in Table 4 . The multivariate analyses have shown that GNRI (HR: 0.973; 95\%CI $0.964-0.982 ; \mathrm{p}<0.001$ ), PNI (HR 0.967; 95\%CI 0.947$0.988 ; \mathrm{p}<0.001)$, CONUT score (HR 1.527; 95\%CI 1.404$1.661 ; \mathrm{p}<0.001)$, and BMI (HR 0.818; 95\%CI 0.767-0.872; $\mathrm{p}<0.001)$ were independent predictors of 5-year mortality in patients with NSTEMI.

Table 3. Baseline laboratory and echocardiography parameters of the study population.

\begin{tabular}{l|c|c|c} 
& $\begin{array}{c}\text { Survivor Group } \\
(\mathrm{n}=736)\end{array}$ & $\begin{array}{c}\text { Nonsurvivor Group } \\
(\mathrm{n}=179)\end{array}$ & $\mathrm{p}$ \\
\hline Albumin $(\mathrm{g} / \mathrm{mL})$ & $4.1 \pm 0.58$ & $3.9 \pm 0.32$ & $<0.001$ \\
\hline Total protein (g/mL) & $7.6 \pm 0.6$ & $7.5 \pm 0.30$ & 0.104 \\
\hline Total cholesterol (mg/dL) median (25th-75th) & $183(155-210)$ & $182(163-207)$ & 0.072 \\
\hline LDL-C (mg/dL) median (25th-75th) & $123(99-145)$ & $122(106-138)$ & 0.602 \\
\hline HDL-C (mg/dL) median (25th-75th) & $39(34.2-47)$ & $40.0(35.0-45.0)$ & 0.476 \\
\hline White blood count (x103) & $8.2 \pm 3.2$ & $9.2 \pm 2.7$ & 0.458 \\
\hline Platelet count (x103) & $245 \pm 65$ & $233 \pm 91$ & 0.053 \\
\hline Hemoglobin (mg/dL) & $13.8 \pm 1.7$ & $13.7 \pm 2.1$ & 0.322 \\
\hline CRP (mg/dL) median (25th-75th) & $0.7(0.3-1.50)$ & $1.0(0.6-1.60)$ & 0.558 \\
\hline Creatine median (25th-75th) & $0.8(0.7-1.0)$ & $0.9(0.7-1.1)$ & 0.581 \\
\hline Urea (mg/dL) median (25th-75th) & $29.9(23.5-36.4)$ & $38.5(29.9-51.3)$ & 0.604 \\
\hline LVEF, \% & $49.7 \pm 6.1$ & $48.7 \pm 9.3$ & 0.059 \\
\hline PNI, mean & $53.2 \pm 7.4$ & $47.9 \pm 6.7$ & $<0.001$ \\
\hline GNRI, mean & $117 \pm 13.8$ & $104 \pm 15.2$ & $<0.001$ \\
\hline CONUT score, median (25th-75th) & $3.0(2.0-3.0)$ & $4.0(3.0-5.0)$ & $<0.001$ \\
\hline
\end{tabular}

LDL-C: low-density lipoprotein cholesterol; HDL-C: high-density lipoprotein cholesterol; CRP: C reactive protein; LVEF: left ventricular ejection fraction; PNI: prognostic nutritional index; GNRI: geriatric nutritional risk index; CONUT: controlling nutritional status. Statistically significant values are given in bold. 

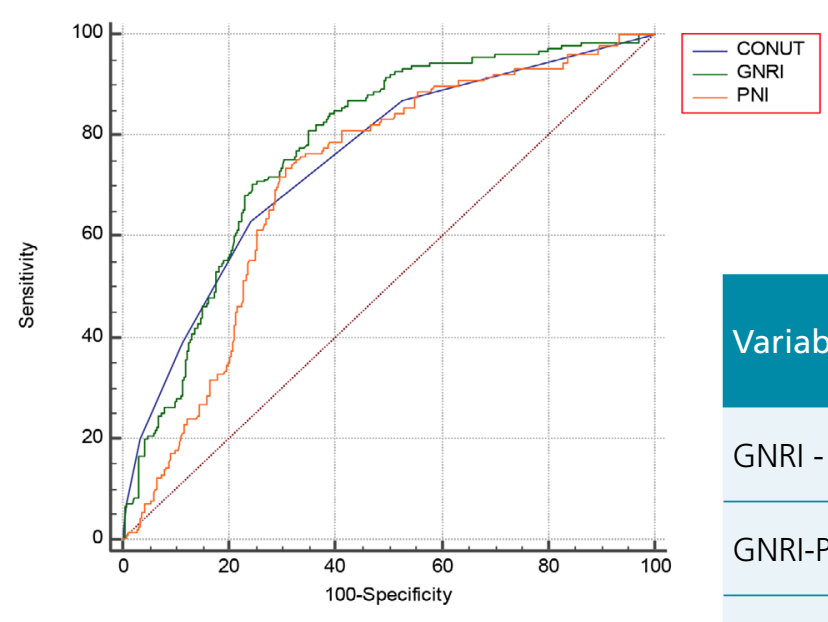

\begin{tabular}{l|c|c|c} 
Variable & AUC & SE & $95 \% \mathrm{Cl}$ \\
\hline GNRI & 0.778 & 0.018 & $0.750-0.805$ \\
\hline CONUT & 0.751 & 0.019 & $0.722-0.779$ \\
\hline PNI & 0.714 & 0.020 & $0.684-0.744$ \\
\hline
\end{tabular}

\begin{tabular}{l|c|c|c|c|c} 
Variable & $\begin{array}{c}\text { Differences } \\
\text { between } \\
\text { areas }\end{array}$ & SE & $95 \% \mathrm{Cl}$ & $\begin{array}{c}\mathrm{Z} \\
\text { statistic }\end{array}$ & $\mathrm{P}$ \\
\hline GNRI - CONUT & 0.027 & 0.0272 & $\begin{array}{c}-0.0264- \\
0.0804\end{array}$ & 0.992 & 0.321 \\
\hline GNRI-PNI & 0.0635 & 0.0238 & $\begin{array}{c}0.0169- \\
0.1100\end{array}$ & 2.672 & 0.0075 \\
\hline PNI-CONUT & 0.0365 & 0.0200 & $\begin{array}{c}-.0002- \\
0.0756\end{array}$ & 1.827 & 0.0677 \\
\hline
\end{tabular}

AUC: area under the curve; Cl: confidence interval; CONUT: controlling nutritional status; GNRI: geriatric nutritional risk index; PNI: prognostic nutritional index; SE: standard error.

Figure 1. Comparison of receiver operating characteristic (ROC) curves for all-cause mortality.



(A) GNRI, geriatric nutritional index.

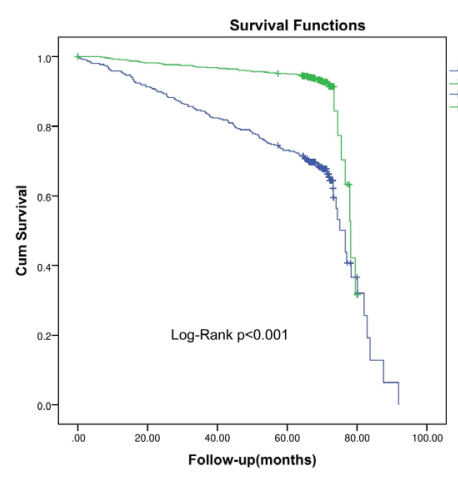

(B) PNI, prognostic nutritional index.

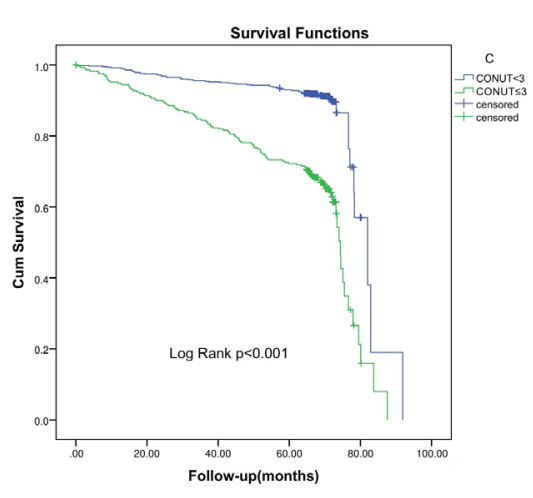

(C) CONUT, controlling nutritional status score.

Figure 2. The Kaplan-Meier analysis for all-cause mortality, according to the cut-off values of (A) GNRI, (B) PNI, and (C) CONUT scores.

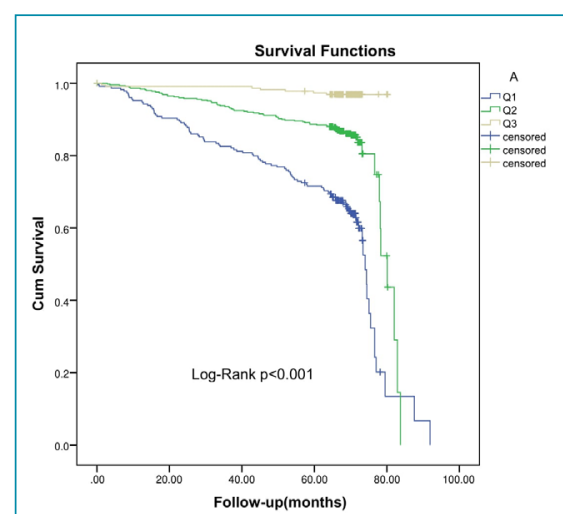

(A) GNRI, geriatric nutritional index.

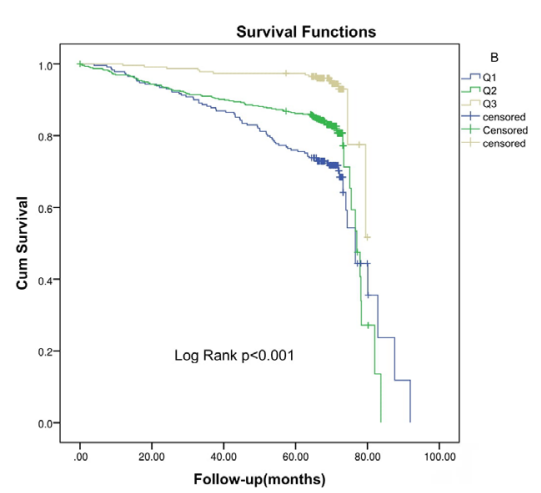

(B) PNI, prognostic nutritional index.

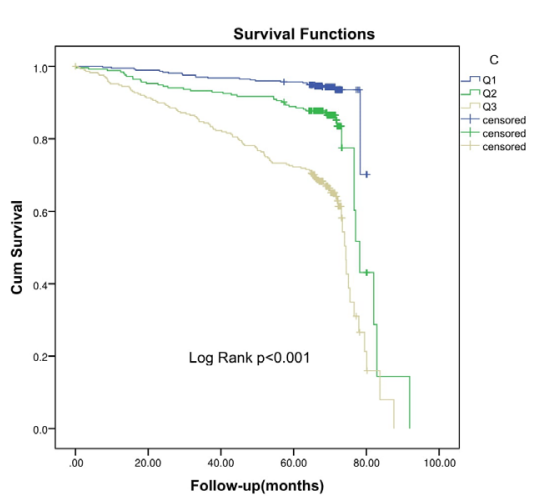

(C) CONUT, controlling nutritional status score.

Figure 3. The Kaplan-Meier analysis for all-cause mortality, according to interquartile of (A) GNRI, (B) PNI, and (C) CONUT scores. 
Table 4. Univariate and multivariate Cox proportional hazard analysis of all-cause mortality.

\begin{tabular}{l|c|c|c|c}
\multirow{2}{*}{ Analysis } & \multicolumn{3}{|c|}{ Univariate } & \multicolumn{2}{c}{ Multivariate } \\
\cline { 2 - 5 } Age & $\mathrm{p}$ & $\mathrm{HR}[95 \% \mathrm{Cl}]$ & $\mathrm{p}$ & $\mathrm{HR}[95 \% \mathrm{Cl}]$ \\
\hline Height & 0.212 & $1.011(0.994-1.029)$ & & \\
\hline Weight & $<0.001$ & $1.134(1.090-1.179)$ & & \\
\hline BMI & 0.131 & $0.985(0.966-1.004)$ & & $0.818(0.767-0.872)$ \\
\hline Albumin & $<0.001$ & $0.787(739-839)$ & $<0.001$ & \\
\hline SBP & 0.001 & $0.639(0.495-0.825)$ & & \\
\hline Total cholesterol & 0.003 & $1.012(1.004-1.019)$ & & \\
\hline Total protein & 0.050 & $1.003(1.00-1.006)$ & & \\
\hline LVEF & 0.079 & $0.800(0.624-1.026)$ & & $0.967(0.947-0.988)$ \\
\hline PNI & 0.089 & $0.982(0.963-1.003)$ & & $1.527(1.404-1.661)$ \\
\hline CONUT score & $<0.001$ & $0.927(0.910-0.945)$ & 0.002 & $0.973(0.964-0.982)$ \\
\hline GNRI & $<0.001$ & $1.494(1.380-1.619)$ & $<0.001$ & $<0.001$ \\
\hline
\end{tabular}

BMI: body mass index; Cl: confidential interval; CONUT: controlling nutritional status; EDV: end-diastolic volume, ESV: end-systolic volume; HR: hazard ratio; GNRI: geriatric nutritional risk index; PNI: prognostic nutritional index; LVEF: left ventricular ejection fraction; SBP: systolic blood pressure. Statistically significant values are given in bold.

\section{DISCUSSION}

This study was the first to examine the association of the CONUT, GNRI, and PNI scores with the long-term mortality in patients with NSTEMI who underwent PCI. It was noted that all the three indexes were significantly associated with the long-term all-cause mortality in patients involved in this study. Besides, in the comparison of predicting mortality, GNRI yielded similar results to CONUT score but was better than PNI. Malnutrition is a frequent and significant problem and is seen especially in hospitalized elderly patients. Earlier reports suggest that malnutrition is closely related to poor prognosis and mortality in terminal kidney failure, malignancy, and hematological diseases ${ }^{6-8}$. In search of a valuable marker, various nutritional indicators have been identified, including lymphocyte count, serum albumin levels, serum cholesterol levels, Mini-Nutritional Assessment (MNA) ${ }^{14}$, and Subjective Global Assessment (SGA) in addition to the CONUT scores, GNRI, and PNI. Among these indicators, the ones with additional parameters put by the healthcare professionals, such as MNA and SGA, although expected to be more accurate, may be considered as potentially biased. On the other hand, CONUT scores, GNRI, and PNI include the objective quantitative data of the patient. In the calculation of the GNRI, only the height, weight, and serum albumin data of the patient are required. Similarly, the calculation of the PNI needs only the serum albumin level and the lymphocyte count of the patient. The calculation of the CONUT score requires the serum cholesterol level of the patient in addition to their serum albumin level and the lymphocyte count. These three indexes use different methods and variables and therefore have unique advantages and disadvantages. Nevertheless, serum albumin is the only common parameter, and its low levels alter the results of all the three indicators. A recent study suggested that in patients who underwent PCI, low serum albumin levels, independent of the traditional risk factors, were associated with major adverse cardiac event (MACE) development ${ }^{15}$. The association of the CONUT scores, GNRI, and PNI with the mortality in patients with $\mathrm{AMI}^{16}$, cardiac failure ${ }^{17}$, and chronic coronary syndrome who underwent elective $\mathrm{PCI}^{18}$ was reported in earlier studies. In a retrospective study, including patients who underwent PCI due to stable coronary disease, the mean follow-up duration was 7.4 years and a high CONUT score was found to be associated with all-cause mortality and nonfatal MI in the longterm follow-up ${ }^{19}$. A research conducted in 802 patients who received elective PCI to the de novo lesions due to stable angina pectoris or objective ischemia showed a significant association between GNRI and poor cardiac prognosis following $\mathrm{PCI}^{20}$. Furthermore, the majority of the patients with chronic coronary syndrome were on an antilipidemic medication; the use of CONUT score on this particular group may seem erroneous. In this study, a low percentage of the patients were on statins, and the difference between the two groups was insignificant. Therefore, the CONUT scores in the study might be considered optimal ${ }^{20}$. In a recent report conducted on 2853 patients with first PCI procedure, 849 had acute coronary syndrome, 
suggesting that low GNRI scores were independent predictors of all-cause mortality ${ }^{21}$.

Moreover, these indicators have also been shown to yield different associations with poor prognosis and mortality in similar disease groups. In a study examining the prognostic values of CONUT score and PNI, conducted in 945 elderly STEMI patients who underwent $\mathrm{PCI}$, at the end of a 2-year follow-up, CONUT scores were found to be associated with the increase in all-cause mortality rates, whereas PNI scores failed to present a predictive value ${ }^{16}$. Conversely, in a research carried out in 345 STEMI patients who underwent primary PCI assessing PNI predictivity only, it was found that PNI was an independent predictor of mortality in this group ${ }^{22}$. Similarly, in a study of 1823 STEMI cases who underwent primary PCI, it was proposed that low PNI values were associated with both in-hospital and 3-year long-term mortality ${ }^{18}$. In a study of 2251 patients, 975 had STEMI and 1276 had NSTEMI, and low GNRI scores were found to be significantly associated with post-MI complications and in-hospital mortality ${ }^{23}$.

In the present study, low GNRI and PNI and high CONUT values were shown to predict the long-term mortality in NSTEMI patients. The multivariate analysis revealed that all the three indicators had independent prognostic values for mortality. Moreover, GNRI was demonstrated to have similar value compared with CONUT score and better than PNI. Since the serum albumin level is the common parameter, the different results were based on serum cholesterol levels and weight data. Lowering the cholesterol levels of the patients is one of the primary goals in coronary heart disease $\mathrm{e}^{24}$. Low cholesterol levels before treatment in a patient attach particular importance, since the nutritional assessment will result in low nutrition status. According to the guidelines, patients with NSTEMI should receive antilipidemics even with low cholesterol levels ${ }^{24}$. However, the possibility of a poorer prognosis of the patients with low cholesterol levels at the time of the index event should always be noted. In the same manner, this study has revealed that low weight was associated with the long-term outcomes. Weight lower than the ideal is associated with fragility, which was reported as closely linked with the long-term poor prognosis in patients with cardiovascular diseases ${ }^{25}$. In patients with coronary heart diseases, overweight or underweight, the latter pointing fragility, both are undesirable. In this study, it was shown that the patients with low GNRI levels, indicating more fragility, were reported to have a higher mortality rate. In NSTEMI cases, the possibility of experiencing the long-term poor prognosis for patients with low weight at the time of index event should be considered.

\section{Limitation}

There were significant limitations to this study. This study was a single-centered retrospective study. Moreover, all-cause mortality was set as the primary end point and the effect of the indicators on cardiovascular-related mortality was not assessed exclusively. Moreover, not all AMI cases were included in this study. The aim of not involving STEMI patients was to form a relatively homogeneous study group due to the differences in the mean age, risk factors, and the comorbidities of the STEMI patients compared with the NSTEMI population. Larger study populations, including all AMI patients assessing the nutritional indicators, are required.

\section{CONCLUSION}

In this study, it was shown that CONUT score, GNRI, and PNI were associated with the long-term mortality in NSTEMI patients who underwent PCI. Furthermore, in the comparison of predicting mortality, GNRI yielded similar results to CONUT score but was better than PNI.

\section{ETHICAL APPROVAL}

All the procedures performed in this study involving human participants were in accordance with the ethical standards of the Institutional and/or National Research Committee and with the 1964 Helsinki declaration and its later amendments or comparable ethical standards. Informed consent was obtained from all the individual participants included in this study.

\section{AUTHORS" CONTRIBUTIONS}

AY: Conceptualization, Investigation, Writing - Review \& Editing. MK: Data Curation, Writing - Original Draft. NYK: Formal Analysis, Software. YC: Methodology, Validation. MCB: Project Administration, Visualization. SK: Resources, Supervision.

\section{REFERENCES}

1. Yeh RW, Sidney S, Chandra M, Sorel M, Selby JV, Go AS. Population trends in the incidence and outcomes of acute myocardial infarction. N Engl J Med. 2010;362(23):2155-65. https://doi.org/10.1056/NEJMoa0908610

2. Terkelsen CJ, Lassen JF, Nørgaard BL, Gerdes JC, Jensen T, Gøtzsche LB-H, et al. Mortality rates in patients with st-elevation vs. Non-st-elevation acute myocardial infarction: Observations from an unselected cohort. Eur Heart J. 2005;26(1):18-26. https://doi.org/10.1093/eurheartj/ehi002

3. Cernea S. Nutritional status and clinical outcomes of cardiac patients in acute settings. Journal of Cardiovascular Emergencies. 2018;4(1):5-7. https://doi.org/10.2478/jce-2018-0007 
4. Pirlich M, Schütz T, Norman K, Gastell S, Lübke HJ, Bischoff SC, et al. The german hospital malnutrition study. Clin Nutr. 2006;25(4):563-72. https://doi.org/10.1016/J.Clnu.2006.03.005

5. Correia MIT, Perman MI, Waitzberg DL. Hospital malnutrition in latin america: A systematic review. Clin Nutr. 2017;36(4):95867. https://doi.org/10.1016/j.clnu.2016.06.025

6. Okamoto S, Ureshino H, Kidoguchi K, Kusaba K, Kizuka-Sano $\mathrm{H}$, Sano $\mathrm{H}$, et al. Clinical impact of the conut score in patients with multiple myeloma. Ann Hematol. 2020;99(1):113-9. https://doi.org/10.1007/s00277-019-03844-2

7. Migita K, Matsumoto S, Wakatsuki K, Ito M, Kunishige T, Nakade $\mathrm{H}$, et al. The prognostic significance of the geriatric nutritional risk index in patients with esophageal squamous cell carcinoma. Nutr Cancer. 2018;70(8):1237-45. https://doi. org/10.1080/01635581.2018.1512640

8. Takahashi $H$, Ito $Y$, Ishii $H$, Aoyama T, Kamoi D, Kasuga $\mathrm{H}$, et al. Geriatric nutritional risk index accurately predicts cardiovascular mortality in incident hemodialysis patients. J Cardiol. 2014;64(1):32-6. https://doi.org/10.1016/j. jjcc.2013.10.018

9. Vickers NJ. Animal Communication: When I'm Calling You, Will You Answer Too? Curr Biol. 2017;27(14):R713-5. https:// doi.org/10.1016/j.cub.2017.05.064

10. Rus VA, Chitu M, Cernea S, Benedek I, Hodas R, Zavate R, et al. Altered nutritional status, inflammation and systemic vulnerability in patients with acute myocardial infarction undergoing percutaneous coronary revascularisation: A prospective study in a level 3 cardiac critical care unit. Nutr Diet. 2020;77(2):212-22. https://doi.org/10.1111/17470080.12536

11. Keskin M, Hayıroğlu M, Keskin T, Kaya A, Tatlısu M, Altay S, et al. A novel and useful predictive indicator of prognosis in st-segment elevation myocardial infarction, the prognostic nutritional index. Nutr Metab Cardiovasc Dis. 2017;27(5):43846. https://doi.org/10.1016/j.numecd.2017.01.005

12. Neumann F, Sousa-Uva M, Ahlsson A, Alfonso F, Banning A, Benedetto U, et al. Esc scientific document group. 2018 ESC/ EACTS guidelines on myocardial revascularization. Eur Heart J. 2019;40(2):87-165. https://doi.org/10.1093/eurheartj/ehy394

13. Thygesen K, Alpert JS, Jaffe AS, Chaitman BR, Bax JJ, Morrow DA, et al. Fourth universal definition of myocardial infarction (2018). J Am Coll Cardiol. 2018;72(18):2231-64. https://doi. org/10.1016/j.jacc.2018.08.1038

14. Guigoz Y, Vellas B, Garry PJ. Assessing the nutritional status of the elderly: The mini nutritional assessment as part of the geriatric evaluation. Nutr Rev. 1996;54(1 Pt 2):S59-65. https:// doi.org/10.1111/j.1753-4887.1996.tb03793.x

15. Wada H, Dohi T, Miyauchi K, Shitara J, Endo H, Doi S, et al. Impact of serum albumin levels on long-term outcomes in patients undergoing percutaneous coronary intervention.
Heart Vessels. 2017;32(9):1085-92. https://doi.org/10.1007/ s00380-017-0981-8

16. Basta G, Chatzianagnostou K, Paradossi U, Botto N, Del Turco $\mathrm{S}$, Taddei $\mathrm{A}$, et al. The prognostic impact of objective nutritional indices in elderly patients with st-elevation myocardial infarction undergoing primary coronary intervention. Int J Cardiol. 2016;221:987-92. https://doi.org/10.1016/j.ijcard.2016.07.039

17. Yoshihisa A, Kanno Y, Watanabe S, Yokokawa T, Abe S, Miyata $M$, et al. Impact of nutritional indices on mortality in patients with heart failure. Open Heart. 2018;5(1):e000730. https:// doi.org/10.1136/openhrt-2017-000730

18. Wada H, Dohi T, Miyauchi K, Endo H, Tsuboi S, Ogita M, et al. Combined effect of nutritional status on long-term outcomes in patients with coronary artery disease undergoing percutaneous coronary intervention. Heart Vessels. 2018;33(12):1445-52. https://doi.org/10.1007/s00380-018-1201-x

19. Wada H, Dohi T, Miyauchi K, Doi S, Konishi H, Naito R, et al. Prognostic impact of nutritional status assessed by the controlling nutritional status score in patients with stable coronary artery disease undergoing percutaneous coronary intervention. Clin Res Cardiol. 2017;106(11):875-83. https:// doi.org/10.1007/s00392-017-1132-z

20. Kunimura A, Ishii H, Uetani T, Aoki T, Harada K, Hirayama K, et al. Impact of geriatric nutritional risk index on cardiovascular outcomes in patients with stable coronary artery disease. Clin Res Cardiol. 2017;106(11):875-83. https://doi.org/10.1007/ s00392-017-1132-z

21. Vikenes K, Andersen KS, Melberg T, Farstad M, Nordrehaug JE. Long-term prognostic value of cardiac troponin $i$ and $t$ versus creatine kinase-mb mass after cardiac surgery in low-risk patients with stable symptoms. Am J Cardiol. 2010;106(6):780-6. https://doi.org/10.1016/j.amjcard.2010.04.038

22. Chen Q-J, Qu H-J, Li D-Z, Li X-M, Zhu J-J, Xiang Y, et al. Prognostic nutritional index predicts clinical outcome in patients with acute st-segment elevation myocardial infarction undergoing primary percutaneous coronary intervention. Sci Rep. 2017;7(1):3285. https://doi.org/10.1038/s41598-017-03364-X

23. Yoo SH, Kook HY, Hong YJ, Kim JH, Ahn Y, Jeong MH. Influence of undernutrition at admission on clinical outcomes in patients with acute myocardial infarction. J Cardiol. 2017;69(3):55560. https://doi.org/10.1016/j.jjcc.2016.05.009

24. Knuuti J, Wijns W, Saraste A, Capodanno D, Barbato E, FunckBrentano C, et al. 2019 esc guidelines for the diagnosis and management of chronic coronary syndromes: The task force for the diagnosis and management of chronic coronary syndromes of the european society of cardiology (esc). Eur Heart J. 2020;41:40777. https://doi.org/10.1093/eurheartj/ehz425

25. Klein BE, Klein R, Knudtson MD, Lee KE. Frailty, morbidity and survival. Arch Gerontol Geriatr. 2005;41(2):141-9. https://doi. org/10.1016/j.archger.2005.01.002 